\title{
ECOLOGY
}

\section{Temperature-dependent transmission of Candidatus Phytoplasma asteris by the vector leafhopper Macrosteles quadripunctulatus Kirschbaum}

\author{
F. Maggi, ${ }^{1,2}$ L. Galetto, ${ }^{3}$ C. Marzachì, ${ }^{3}$ D. Bosco ${ }^{4}$ \\ 'School of Civil Engineering, University of Sydney, Australia; ${ }^{2}$ The ISI Foundation, Turin, Italy; \\ ${ }^{3}$ Istituto per la Protezione Sostenibile delle Piante, IPSP, CNR, Turin, Italy; ${ }^{4}$ DISAFA, University of \\ Turin, Italy
}

\begin{abstract}
A set of experiments was carried out to characterize how temperature affects the spread of chrysanthemum yellows phytoplasma (CYP), a strain of Candidatus Phytoplasma asteris, in Chrysanthemum carinatum plants transmitted by the Macrosteles quadripunctulatus leafhopper. Experiments provided data on CYP latency period in insect and plant host, $M$. quadripunctulatus adult mortality rate, and epidemics progression in plants under controlled conditions inside climatic chambers. Experiments were conducted at temperatures ranging between 15 and $30^{\circ} \mathrm{C}$. Empirical laws for temperature-dependent epidemiological parameters were next derived and used in a dynamical model of the epidemics progression. Experiments showed that CYP epidemics was faster at higher temperatures and the model could
\end{abstract}

Correspondence: Federico Maggi, The University of Sydney, School of Civil Engineering, 2006 Sydney, NSW, Australia.

Tel.: +61.293.512.222.

E-mail: federico.maggi@sydney.edu.au

Key words: Aster yellows, phytoplasma vector, epidemics, phytoplasma, temperature, modeling.

Contributions: FM performed the whole mathematical and modeling work and wrote the manuscript (together with DB); LG performed the transmission experiments and some of the molecular analyses; $\mathrm{CM}$ performed molecular analyses and revised the manuscript; DB planned the experiments and wrote the manuscript (together with FM).

Acknowledgements: the authors would thank two anonymous Reviewers fro their constructive comments. This work was funded by Regione Piemonte, Italy, under the grant CIPE Adoption of a multidisciplinary approach to study the grapevine agroecosystem: analysis of biotic and abiotic factors able to influence yield and quality.

Received for publication: 7 March 2014.

Revision received: 17 June 2014.

Accepted for publication: 19 June 2014.

This work is licensed under a Creative Commons Attribution NonCommercial 3.0 License (CC BY-NC 3.0).

(C) Copyright F. Maggi et al., 2014

Licensee PAGEPress, Italy

Entomologia 2014; 2:202

doi:10.4081/entomologia.2014.202 replicate these observations with relatively high accuracy (correlation $>98.03 \%$ and residuals <14.5\%). The epidemics spreading rate increased linearly from 0.2 plants infected per day at $15^{\circ} \mathrm{C}$ to about 0.7 plants per day at $30^{\circ} \mathrm{C}$, possibly due to: i) faster CYP multiplication in the host plants and ii) higher frequency of feeding bouts of $M$. quadripunctulatus at higher temperatures.

\section{Introduction}

Phytoplasmas are mollicutes (wall-less bacteria) associated with diseases of many cultivated and wild plant species worldwide (Foissac \& Wilson, 2010). They are phloem-restricted pathogens found as pleomorphic bodies in the sieve tubes of infected plants (Lee et al., 2000) and transmitted by phloem-sucking leafhoppers, planthoppers and psyllids (Weintraub \& Beanland, 2006). Phytoplasmas are obligatory parasites of both plants and insects, and their spread depends on the presence of vector insects and/or infected vegetative propagation materials. Phytoplasmas are transmitted in a persistent manner: after an acquisition access feeding on infected plants, a latent period (during which phytoplasmas multiply in the midgut, hemocytes, and salivary glands, and possibly in other insect organs) is needed before the insect becomes infective. Once the latent period is completed, the vector remains infective for life (Marzachì et al., 2004). Whereas phytovirus epidemics have been studied in detail both experimentally and theoretically (e.g., Ferriss \& Berger, 1993; Jeger et al., 1998; Madden et al., 2000), only basic mechanisms of phytoplasma transmissions have been investigated (e.g., Weintraub \& Beanland, 2006; Bosco \& D'Amelio, 2010) while the dynamics of phytoplasma epidemics as-awhole still remain largely unreferenced. A mollicute epidemiological model was developed for the corn stunt in Central America (Vandermeer \& Power, 1990), a disease associated with Spiroplasma kunkelii, but this model only included basic characteristics of pathogen dynamics. Generic epidemics models exist in great number and could potentially be used with CYP epidemics, but values of parameters specific to host plant and vector insect are currently missing, as well as their relationship with the temperature.

Since phytoplasma-associated diseases cause heavy damages to important crops such as grapevine, coconut, pome, stone fruits, horticultural and ornamental, understanding the response of phytoplasma epidemics to environmental factors such as temperature and climatic variability can represent a useful tool to comprehend the way epidemics approach an equilibrium, the conditions under which they persist or vanish (Van Maanen \& $\mathrm{Xu}, 2003$ ) and, ultimately, the features that can be used to improve effectiveness of control operations. It is 
widely known that the metabolism of insects, plants and phytoplasmas is influenced by the temperature, hence also the epidemics spreading rate should depend to some extent on temperature. To date, however, no studies have linked changes of phytoplasma disease progression to changes in climatic conditions (Foissac \& Wilson, 2010), but a recent contribution (Salar et al., 2013) showed that the multiplication kinetics of Flavescence dorée phytoplasma is temperature-dependent. Specifically, predicting progression curves of phytoplasma diseases under scenarios of changing environment temperature represents an important capability to cope with projections of global warming and climate change (IPCC, 2008).

The aim of this work is to assess the temperature dependence of i) the CYP latency period in the host plant $C$. carinatum and leafhopper vector $M$. quadripunctulatus; ii) the epidemics progression in a fully controlled CYP - C. carinatum - M. quadripunctulatus patho-system model in climatic chamber; and iii) describe with a mathematical model the temperature-dependent epidemics progression using empirically-based parameter sensitivity to temperature.

The experiments focused on a well characterized patho-system model represented by the host-plant $C$. carinatum (Schousboe), the chrysanthemum yellows phytoplasma (CYP), and its leafhopper vector M. quadripunctulatus (Kirschbaum). M. quadripunctulatus is a multivoltine leafhopper of the family Cicadellidae, subfamily Deltocephalinae, polyphagous on mono- and dicotyledonous herbaceous plants, and well known vector of phytoplasmas of the Candidatus Phytoplasma asteris species. Several other congeneric species are known as phytoplasma vectors as well, making these leafhoppers among the most common vectors of phytoplasma worldwide (Weintraub $\&$ Beanland, 2006). Due to its fast incubation in plants, short latency in vectors, rapid development of detectable symptoms, and extremely high transmission efficiency (Palermo et al., 2001; Saracco et al., 2006; Bosco et al., 2007), the CYP-M. quadripunctulatus-daisy plants system represents an optimal model system for the investigation of temperature dependent epidemiological parameters. Epidemiological parameters of $C$. carinatum and $M$. quadripunctulatus were obtained in experiments at various constant temperatures ranging between $15^{\circ} \mathrm{C}$ and $30^{\circ} \mathrm{C}$ in fully-controlled climatic chamber experiments. Experiments allowed us to establish empirical laws for temperature dependent-epidemiological parameters that are eventually aimed to be used in a mechanistic model of CYP epidemics in plants.

\section{Materials and Methods}

\section{Chrysanthemum yellows phytoplasma, vector insect and plant host}

CYP is a strain of the Candidatus Phytoplasma asteris phytoplasma (16Sr-IB) (Lee et al., 2004) that has been isolated in the field from Argyranthemum frutescens (L.) Schultz-Bip. In the Italian Riviera, CYP can be transmitted by several species of leafhoppers and can infect a variety of dicotyledonous plants (Conti et al., 1988; Palermo et al., 2001; Bosco et al., 2007).

Healthy colonies of $M$. quadripunctulatus were maintained on oat, Avena sativa $L$., inside plexiglas and nylon cages in growth chambers at $25^{\circ} \mathrm{C}$ under a photoperiod of 16:8 (L:D) h. Daisy test plants were grown from seed, transplanted individually into $8 \times 8 \mathrm{~cm}^{2}$ pots, and exposed to infective leafhoppers when they were $15 \mathrm{~cm}$ high $(40 \mathrm{~d}$ after germination).

The CYP acquisition and transmission pathways between $C$. carinatum and $M$. quadripuctulatus adults are depicted in Figure 1, and represent the conceptual basis for our experimental work and mathematical analysis.

\section{Chrysanthemum yellows phytoplasma latency}

\section{in M. quadripunctulatus}

To measure the CYP latency in the leafhopper (time between the beginning of the acquisition feeding and the effective inoculation feeding), $3^{\text {rd }} 5^{\text {th }}$ instar nymphs of $M$. quadripunctulatus from a healthy colony were caged on CYP-infected daisies for a seven-day acquisition access period (AAP), then transferred in groups of five onto healthy daisies for successive two-day inoculation access periods (IAP) until death. Nymphs were used for acquisition because M. quadripunctulatus adults are relatively short living and, due to the long latency of CYP phytoplasma in the insect, the use of adults for acquisition experiments would lead to a high mortality before the end of the experiment. The latency period was assumed to start from the first day of AAP; but a seven-day acquisition access period was chosen in order to maximize the proportion of insects that acquired CYP. Experiments, including acquisition, latency and all the inoculation periods, were carried out at $15,20,25$ and $30^{\circ} \mathrm{C}$ constant temperatures inside climatic chambers with L16:D8 hour photoperiod, and 70\% relative humidity. After the inoculation, daisies were sprayed with insecticides, transferred in an insect-proof greenhouse, and maintained for one month for visual detection of symptoms.

\section{Chrysanthemum yellows phytoplasma latency in C. carinatum}

To determine the CYP incubation period in the plant (time between the beginning of the inoculation access period (IAP) of the vector and symptom appearance), infectious leafhoppers, fed on CYP-infected daisies and maintained on oat to complete latency until 20 days post acquisition, were transferred in groups of five onto four healthy test plants for a two-day IAP. Test plants were then sprayed with insecticide and maintained for one month under the same conditions. Inoculation and symptom incubation took place at constant $15,20,25$ and $30^{\circ} \mathrm{C}$ temperature. CYP infection in plants was determined by visual inspection for typical symptoms (e.g., distortion of the apex). The time of appearance of symptoms was recorded as the final date of the incubation period in the plant.

\section{Chrysanthemum yellows phytoplasma epidemics in climatic chambers}

In order to observe temperature-dependent CYP epidemics progres-

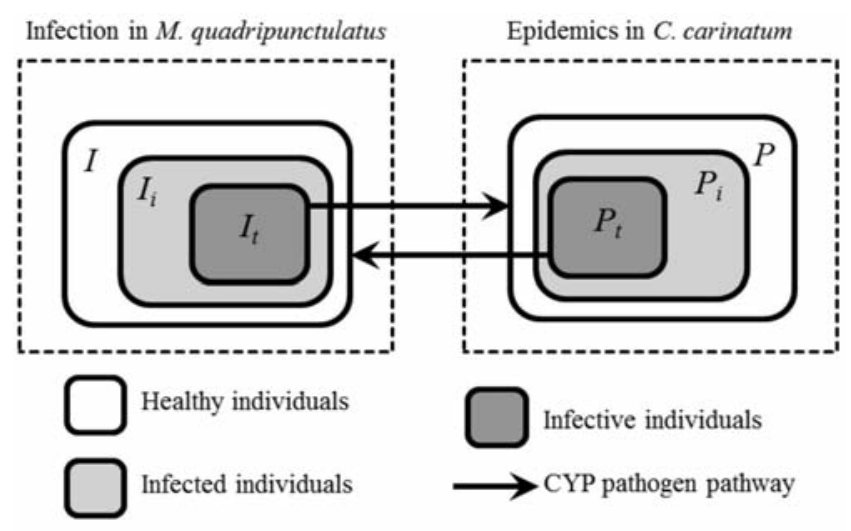

Figure 1. Chrysanthemum yellows phytoplasma (CYP) pathway across insects, $I$, and plants, $P$. The subscripts $i$ and $t$ indicate infected (latent and infective) and infective individuals, while the arrows represent the CYP pathway. 
sion, experiments under controlled conditions were carried out at various constant temperatures inside climatic chambers. Sixteen seedlings of $C$. carinatum individually grown in $8 \times 8 \mathrm{~cm}^{2}$ pots at the sixeight leaf stage (35-40 days after the sowing) were caged inside Plexiglas and nylon isolators of $50 \times 50 \times 40 \mathrm{~cm}^{3}$ inside a climatic chamber (plants were regularly distributed in four rows of four pots). Ten CYP-infective $M$. quadripunctulatus adult leafhoppers were released inside the cages for 22 days. Infective leafhoppers were obtained by caging $3^{\text {rd }} 4^{\text {th }}$ instar nymphs from healthy colonies on CYP-infected source plants for 7 days, transferred to oat plants and emerged as adults during the week before the experiments. Leafhoppers were reared at $25^{\circ} \mathrm{C}$ and, at the beginning of the experiments, they had already completed the latency period since they fed on source plants 20 days before.

Three concomitant sets of experiments were conducted at temperatures of $15^{\circ} \mathrm{C}, 25^{\circ} \mathrm{C}$ and $30^{\circ} \mathrm{C}$ in three different climatic chambers. At all the temperatures, the photoperiod was L16:D8 hours. Cages were isolated in each climatic chamber to reduce disturbances. Two apical leaves of each plant were gently collected with forceps after 7, 11, 16, 22 and 30 days from the beginning of the experiments in order to detect CYP presence. Apical leaves were chosen because they host the higher titer of CYP (Saracco et al., 2006). Total DNA was extracted from these leaves $(0.1 \mathrm{~g}$ tissue) with the PureLink Plant Kit (Invitrogen, Carlsbad, CA, USA) and eluted in $100 \mathrm{~mL}$ of kit elution buffer. Total DNA was extracted with the same protocol from healthy daisies. Two microliters were then used as template in direct PCR. The presence of CYP in inoculated plants was assayed by conventional nested PCR using the R16F2/R2 followed by R16F1/R1 (I) primers (Lee et al., 1993; 1994). Nested PCR was chosen because it represents the most sensitive assay to detect phytoplasmas and is more sensitive than qPCR (Galetto et al., 2005; Roggia et al., 2014). Two microliters of plant DNA were added to a reaction mix containing $200 \mathrm{mM}$ dNTPs, $1.5 \mathrm{mM} \mathrm{MgCl} 2,500 \mathrm{nM}$ of each primer and $0.5 \mathrm{U}$ of Taq DNA polymerase (PolyTaq, Polymed, Sambuca, Italy). Cycling conditions were as detailed in the original papers. The products were separated in a $1 \%$ agarose gel, buffered in TBE $(90 \mathrm{mM}$ Tris-Borate, $2 \mathrm{mM}$ EDTA, pH 8.3), stained with ethidium bromide, and visualized under UV light. At the last sampling date at 22 days, insects that survived were removed and counted, while plants were sprayed with insecticides and transferred to an insect-proof greenhouse for three extra weeks for symptom development. Thirty-six days after the beginning of the experiments symptoms were recorded and infected plants counted.

\section{A model of chrysanthemum yellows phytoplasma epidemics in climatic chambers}

To replicate the experimental observations of CYP spreading in climatic chambers in a mathematical framework, a simple model based on Madden et al. (2000) was elaborated to include CYP-specific transmission features and infective adults of $M$. quadripunctulatus. The model state variables, also represented in Figure 1, are the number of infective $M$. quadripunctulatus adult $I_{t}$, the total number of plants $P$, the number of infected plants Pi (including both latently infected plants with no symptoms and infective plants with symptoms) and the number of infective plant $P_{t}$ (with symptoms). Note that latency in the leafhopper was not taken into consideration because $M$. quadripunctulatus adults were caged in the climatic chambers when the latent period was already completed. The rate of change of these state variables was described by the following ordinary and delay differential equations

$$
\begin{aligned}
& \frac{\mathrm{d} P_{i}(t)}{\mathrm{d} t}=D(T) \beta(T) \frac{P-P_{i}(t)}{P} I_{t}(t) \\
& \frac{\mathrm{d} P_{i}^{*}(t)}{\mathrm{d} t}= \begin{cases}\frac{\mathrm{d} P_{i}\left(t-t_{s}\right)}{\mathrm{d} t} & \text { for } t \geq t_{s}(T) \\
0 & \text { for } t<t_{s}(T)\end{cases}
\end{aligned}
$$

$$
\begin{aligned}
& \frac{\mathrm{d} P_{t}(t)}{\mathrm{d} t}=\left\{\begin{array}{lll}
\frac{\mathrm{d} P_{i}\left(t-L_{P}(T)\right)}{\mathrm{d} t} & \text { for } & t \geq L_{P}(T) \\
0 & \text { for } & t<L_{P}(T)
\end{array}\right. \\
& \frac{\mathrm{d} I_{t}(t)}{\mathrm{d} t}=-\delta(T) I_{t}(t)
\end{aligned}
$$

where $P=16$ is the number of $C$. carinatum seedlings in the cage, $D(T)$ in Eq. (1a) is the insect mobility rate expressing the number of plants visited by one adult per unit time at temperature $T, \beta(T)$ in Eq. (1a) is the probability of CYP transmission at that temperature, $L_{P}(T)$ in Eq. (1c) is the CYP latency in $C$. carinatum after acquisition at temperature $T$, and $\delta(T)$ in Eq. (1d) is the adult $M$. quadripunctulatus mortality rate at temperature $T$. The variable $P_{i}^{*}(t)$ in Eq. (1b) represents the number of plants that have been infected at time $\left(t-t_{s}\right)$, where $t_{S}(T)$ is the minimum detection time (a few days, normally) expressing the time lag required by CYP to reach a concentration of cells per ng of plant DNA large enough (less than 10) to be detected by our PCR method, and is thought to be a function of the temperature. $P_{i}^{*}(t)$ is the variable that is required for comparison with experiments because any infection occurred after time $\left(t-t_{s}\right)$ would not be detected by PCR at time $t$; note that use of $P_{i}(t)$ instead of $P_{i}^{*}(t)$ for comparison would lead to a systematic bias between model calculations and experimental observations.

Eq. (1) can be solved analytically and a short expression valid for $t \geq 0$ is

$$
\begin{aligned}
& P_{i}(t)=P\left(1-e^{-D(T) \beta(T) \frac{I_{t 0}-L_{t}(t)}{\delta(T) P}}\right) \\
& P_{i}^{*}(t)=H\left\{t-t_{s}(T)\right\} \times P_{i}\left(t-t_{s}(T)\right) \\
& P_{i}(t)=H\left\{t-L_{p}(T)\right\} \times P_{i}\left(t-L_{p}(T)\right) \\
& I_{t}(t)=I_{t 0} \mathrm{e}^{-\delta(T) t}
\end{aligned}
$$

where $I_{t 0}=I_{t}(t=0)=10$ is the initial number of $M$. quadripunctulatus adults in the climatic chamber, and $H\{x\}$ is the Heaviside function (i.e., $H\{x\}=1$ for $x \geq 0$ and $H\{x\}=0$ otherwise).

Eq. (2) are used to estimate the values of $\beta, D, t_{s}$ and $\delta$ at different temperatures against experimental data; note however that because $\beta$ and $D$ could not be independently determined, term $D^{\prime}=D \beta$ was introduced, which defines the effective epidemics spreading rate by combining the insect mobility rate $D$ with the transmission probability $\beta$. Estimation of $D^{\prime}, t_{s}$ and $\delta$ was performed against phytotron data sets at 15,25 and $30^{\circ} \mathrm{C}$ by minimizing the distance between observed and modeled values of $P_{i}(t)$ using the Levenberg-Marquardt algorithm implemented in the PEST (Parameter ESTimation) software (Doerthy, 2004).

\section{Goodness of fit}

Experimental data were used to determine empirical scaling laws and dynamic equations to describe each measured quantity as a function of the temperature. To this purpose, a linear regression method (least square fitting) was used to estimate polynomial parameters for $L_{I}(T)$ and $L_{P}(T)$ as a function the temperature, while a nonlinear regression method was used to estimate $D^{\prime}(T), t_{s}(T)$ and $\delta(T)$, which were next described by polynomial functions of $T$. For each estimation instance, goodness of fit was measured by the correlation coefficient $\mathrm{R}=\operatorname{cov}(m, o) / \sigma_{m} \sigma_{o}$ and normalized root mean square error (residual) NRMSE $=\left(\frac{1}{n} \sum_{i=1}^{n}\left(m_{i}-o_{i}\right)^{2}\right)^{1 / 2} /(\max \{o\}-\min \{o\})$ where $m$ and $o$ are the modeling and experimental points, respectively. The values of $\mathrm{R}$ and NRMSE are reported as percent. 


\section{Results}

Experimental results and selected mathematical analyses are presented below.

\section{Temperature-dependent latency}

\section{in M. quadripunctulatus}

The experimental CYP latency periods $L_{I}$ in $M$. quadripunctulatus, calculated from day 1 of the AAP, showed a strong correlation with the experimental temperatures tested here. In particular, Figure 2 suggests that $L_{I}$ decreased linearly from about $40 \mathrm{~d}$ at $15^{\circ} \mathrm{C}$ (from the beginning of the acquisition) to about $30 \mathrm{~d}$ at $20^{\circ} \mathrm{C}$, and remained nearly constant at about $18 \mathrm{~d}$ for $T \geq 25^{\circ} \mathrm{C}$. Because the overall trend was not linear, a piece-wise linear function was used to describe $L_{I}$ as

$$
L_{l}(T)= \begin{cases}a_{I} T+b_{I} & \text { for } \quad T<25^{\circ} C \\ 18 \text { days } & \text { for } T \geq 25^{\circ} C\end{cases}
$$

with $a_{I}=-2.2 \mathrm{~d} /{ }^{\circ} \mathrm{C}$ and $b_{I}=73.3 \mathrm{~d}$ obtained by least square fitting, which resulted in $\mathrm{R}=94.73 \%$ and $\mathrm{NRMSE}=13.41 \%$ (Figure 2). A similar piecewise trend was observed also in Murral et al., (1996) for $M$. quadrilineatus, a closely related species to M. quadripunctulatus used in this study.

\section{Temperature-dependent latency in $C$. carinatum}

The latency time $L_{P}$ in $C$. carinatum was nonlinearly decreasing for $T$ increasing between $15^{\circ} \mathrm{C}$ and $25^{\circ} \mathrm{C}$, while it increased again for $T>25^{\circ} \mathrm{C}$. Experimental data showed that a function could suitably describe the effect of temperature on $L_{P}$ as

$$
L_{P}(\mathrm{~T})=a_{P} T^{2}+b_{P} T+c_{P}
$$

with $a_{P}=0.13 \mathrm{~d} /{ }^{\circ} \mathrm{C}^{2}, b_{P}=-6.13 \mathrm{~d} /{ }^{\circ} \mathrm{C}$, and $c_{P}=85.56 \mathrm{~d}$ obtained by least square fitting with $\mathrm{R}=98.29 \%$ and NRMSE $=6.62 \%$ (Figure 2).

\section{Temperature-dependent chrysanthemum yellows phytoplasma epidemics in climatic chambers}

The time course of infection of $C$. carinatum test plants at the three constant temperatures of 15,25 and $30^{\circ} \mathrm{C}$ showed a substantial dependence on temperature, with the average number of infected plants $P_{i}^{*}$ increasing relatively slowly at $T=15^{\circ} \mathrm{C}$ (Figure 3A) and more rapidly at

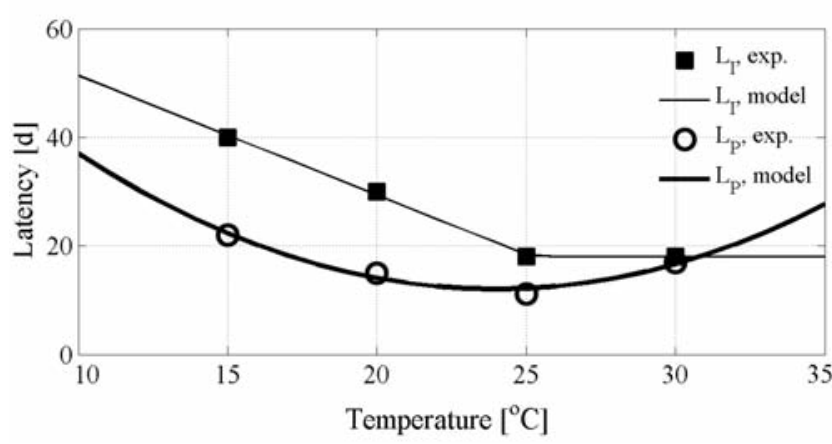

Figure 2. Experimental and modelled latency $L_{I}$ and $L_{P}$ in Macrosteles quadripunctulatus and Chrysanthemum carinatum, respectively, as a function of the temperature $T$.
$T=25^{\circ} \mathrm{C}$ and $T=30^{\circ} \mathrm{C}$ (Figure 3B,C). Adult count showed that from 70 (at 15 and $30^{\circ} \mathrm{C}$ ) to $80 \%$ (at $25^{\circ} \mathrm{C}$ ) of $M$. quadripunctulatus infective adults survived at the end of the experiments, and suggested that temperatures used in the experiments did not impact substantially adult survival over the initial 22 days of adult life. Note however, that this high survival may be explained by adults' age, which were incubated and released into the chambers soon after emergence.

Application of Eq. (2) for the time varying value of $P_{i}^{*}(t)$ with $P=16$ and $I_{t 0}=10$ required estimation of $D^{\prime}, t_{s}$ and $\delta$, while $L_{P}(T)$ was described using Eq. (4). The optimal values of $D^{\prime}, t_{s}$ and $\delta$ at each temperature (Table 1) led to mathematical description of experimental epidemics in plants with relatively high accuracy for the three temperatures tested here (Figure 3 ) as emphasized by the modeled $P_{i}^{*}(t)$ values being nearly always comprised within the experimental standard deviation, and by the high correlation coefficient $\mathrm{R}>98.03 \%$ and small residuals NRMSE $<14.5 \%$ between observed and modeled $P_{i}^{*}$.

The estimated effective spreading rate $D^{\prime}$ and phytoplasma detection time $t_{s}$ strongly depended on temperature. In particular, $D^{\prime}$ increased sharply with $T$ (Table 1). We can hypothesize: that i) $M$. quadripunctulatus may be induced to move more frequently from one
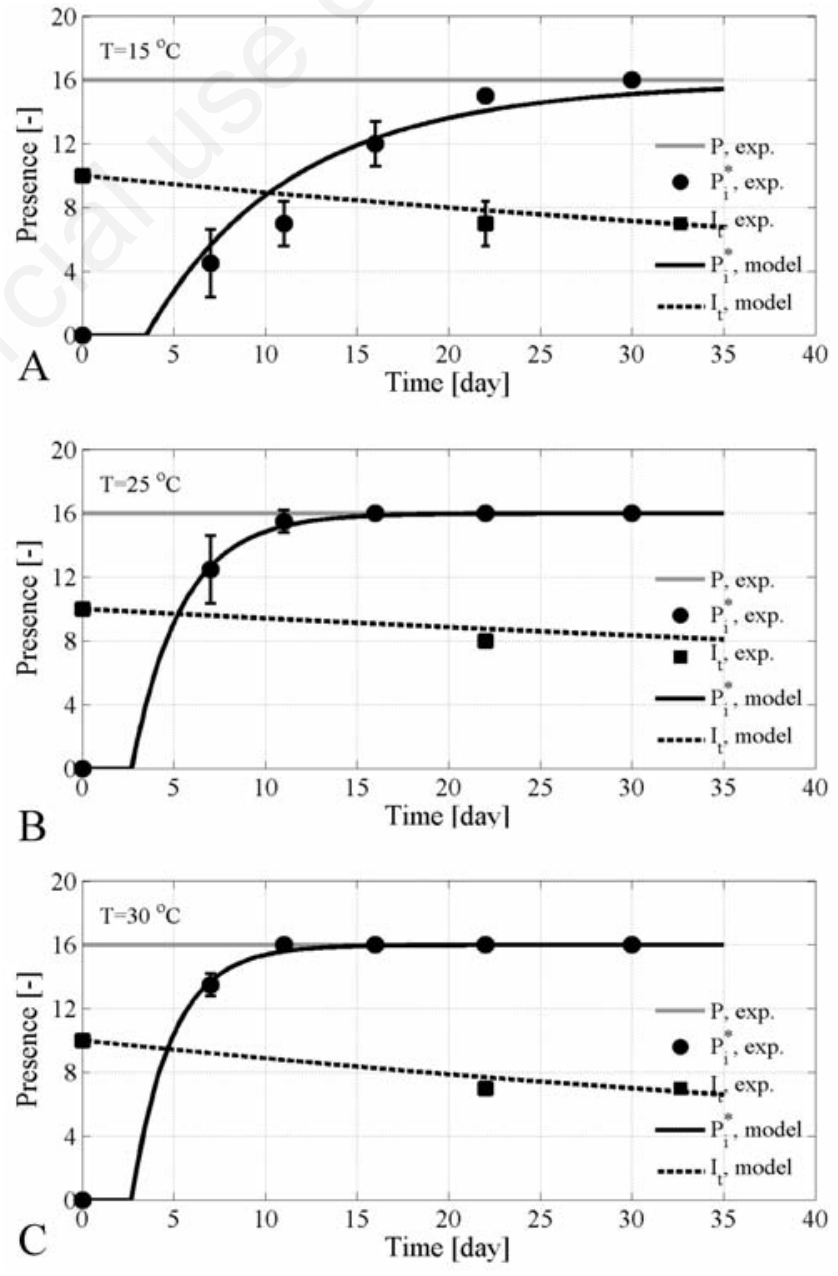

Figure 3. Experimental and modelled $P_{i}^{*}(t)$ number of CYP infected plants (positive at the PCR method) over time describing the epidemics progression at temperatures equal to $15^{\circ} \mathrm{C}(\mathrm{A})$,

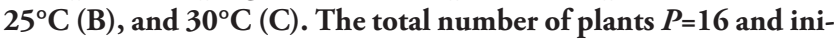
tial insect number $I=I_{t 0}=10$ at time $t=0 \mathrm{~d}$ were used. 
plant to another when exposed to high $T$, ii) CYP transmission efficiency $\beta$ to $C$. carinatum could be high at high $T$, iii) feeding activity increased with temperature, iv) some or all above hypothese occur simultaneously. We noted that the detection time $t_{s}$ decreased for an increasing $T$, thus indicating that CYP pathogen multiplication rate within plants may be faster at higher temperatures (Table 1). This result aligns to the temperature sensitivity of Flavescence dorée phytoplasma observed by Salar et al. (2013).

\section{Temperature-dependent epidemics effective spreading rate}

The modeling results clearly highlight that the epidemics in plants spread progressively faster as the temperature raised from $15^{\circ} \mathrm{C}$ to $30^{\circ} \mathrm{C}$ (Figure 3 ). This characteristic is fully embedded in the parameter $D^{\prime}$, which was shown in Table 1 to change substantially as a function of the $T$.

When reporting $D^{\prime}$ vs $T$, as in Figure 4, one notes that $D^{\prime}$ increased linearly from $0.2 \mathrm{~d}^{-1}$ at $15^{\circ} \mathrm{C}$, to about $0.7 \mathrm{~d}^{-1}$ at $30^{\circ} \mathrm{C}$. Upon this observation, a linear relationship between $D^{\prime}$ and $T$ was used as

$D^{\prime}(T)=a_{D} T+b_{D}$

with $a_{D}=0.036 \mathrm{~d}^{-1}{ }^{\circ} \mathrm{C}^{-1}$ and $b_{D}=-0.335 \mathrm{~d}^{-1}$, which corresponded to $\mathrm{R}=99.8 \%$ and residuals $\mathrm{NRMSE}=2.54 \%$. Eq. (5) is presumably a good approximation of the epidemics effective spreading rate within the temperature range $15^{\circ} \mathrm{C} \leq T \leq 30^{\circ} \mathrm{C}$, but it may introduce a level of uncertainty below $15^{\circ} \mathrm{C}$ and above $30^{\circ} \mathrm{C}$.

Table 1. Summary of parameters estimated against experimental data of chrysanthemum yellows phytoplasma epidemics in Chrysanthemum carinatum transmitted by Macrosteles quadripunctulatus in climatic chambers.

\begin{tabular}{llccc}
\multicolumn{3}{l}{ Parameter } & \multicolumn{3}{c}{$\begin{array}{c}\text { Temperature } \\
{\left[{ }^{\circ} \mathrm{C}\right]}\end{array}$} \\
& & 15 & 25 & 30 \\
$D^{\prime}$ & {$\left[\mathrm{d}^{-1}\right]$} & 0.2 & 0.59 & 0.74 \\
$t_{s}$ & {$[\mathrm{~d}]$} & 3.44 & 2.66 & 2.66 \\
\hline$\delta$ & {$\left[\mathrm{d}^{-1}\right] \times 10^{-2}$} & 1.11 & 0.61 & 1.18 \\
$\mathrm{R}$ & $\%$ & 98.03 & 99.53 & 98.48 \\
\hline NRMSE & $\%$ & 14.5 & 5.0 & 8.9 \\
\hline
\end{tabular}

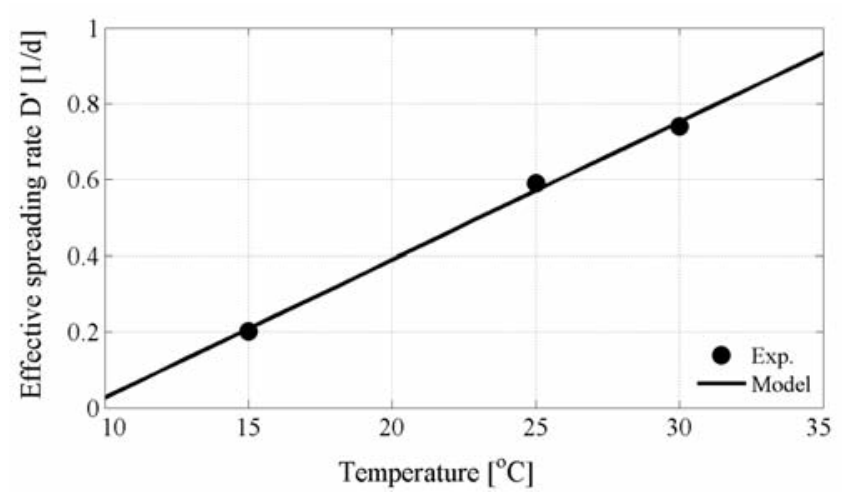

Figure 4. Experimental and modelled epidemic effective spreading rate $D$ ' as a function of the temperature $T$.
Temperature-dependent chrysanthemum yellows phytoplasma detection time

We have previously noted that also the CYP minimum detection time in plant $t_{s}$ by PCR method can vary with $T$. A linear relationship between $t_{s}$ and $T$ is proposed here as

$t_{s}(T)=a_{s} T+b_{s}$

with $a_{s}=-0.055 \mathrm{~d}^{\circ} \mathrm{C}^{-1}$ and $b_{s}=4.22 \mathrm{~d}$, corresponding to $\mathrm{R}=94.5 \%$ and residuals NRMSE $=15.43 \%$ (Figure $5 \mathrm{~A}$ ). Because the detection time is related to the velocity of CYP replication in the host plant, it is expected to be correlated to the latency $L_{P}$ as well. However, a representation of $t_{s}(T)$ of Eq. (6) against $L_{P}(T)$ of Eq. (4) in the temperature continuum shows a relatively important distance from the experimental points (Figure 5B), suggesting that no trivial relation exists between the two quantities. This discrepancy may be related to the different scaling; in fact $L_{P}$ was found to scale as $T^{2}$ while $t_{s}$ was found to scale as $T$ with some residuals at $25^{\circ} \mathrm{C}$ and $30^{\circ} \mathrm{C}$. Therefore, we conclude that no strong evidence is available as yet that CYP minimum detection time is deterministically related to $L_{P}$ in plants and can be explained by a dynamic process of CYP cell multiplication in the host plants not explicitly accounted for in our mathematical approach.

\section{Epidemics sensitivity to the temperature}

As demonstrated by our experiments (Figure 3) and emphasized by mathematical analysis (Figure 4), the epidemics effective spreading rate $D^{\prime}$ strongly depended on $T$, dependence which was described by a
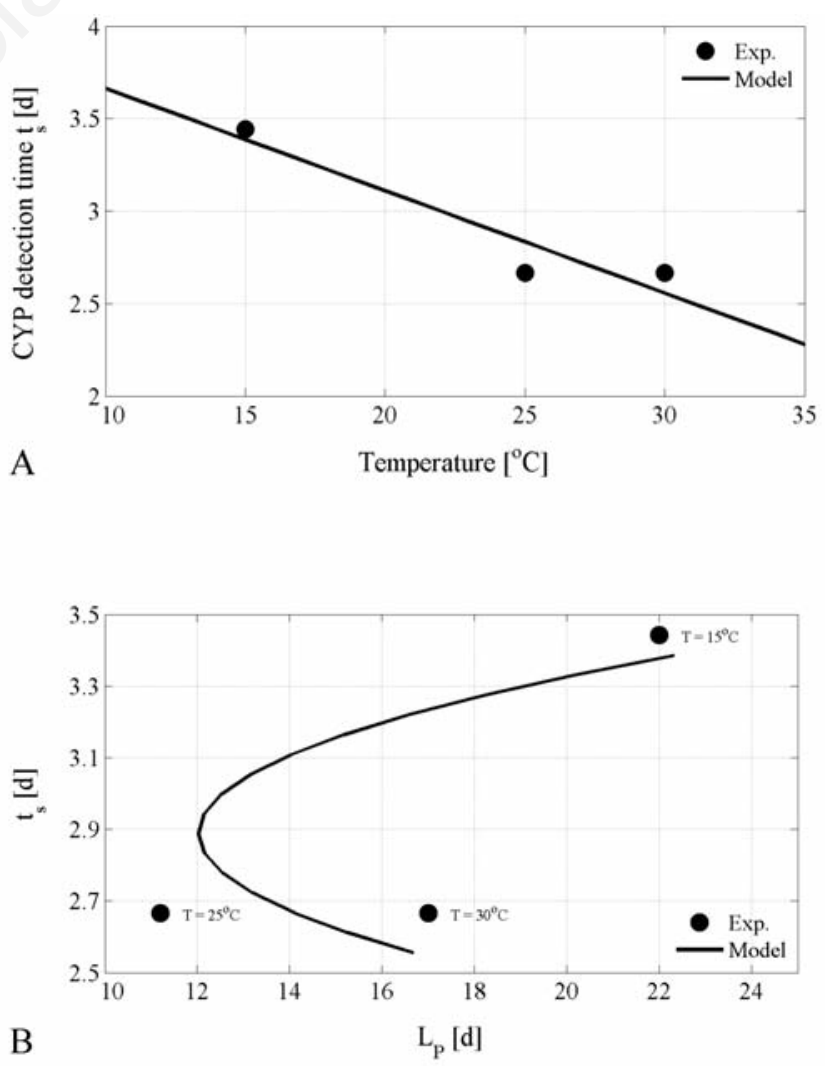

Figure 5. Experimental and modelled CYP detection time (A) as a function of temperature $T$ and $(B)$ as a function of latency in plants $L_{P}(\mathrm{~T})$. 
linear scaling law. Note that also the latency $L_{P}$ was found to be a function of $T$ (Figure 2) and that $L_{P}$ is as important as $D$ ' in determining the epidemics progression. Using the analytical model solution in Eqs. (2), we analyzed the epidemics dynamics within the temperature continuum, where the epidemics progression was measured by the time evolution of infected and infective plants $P_{i}(t)$ and $P_{t}(t)$, respectively. In this analysis, the settings of the controlled experiments were replicated, that is, $P=16$ and $I_{t}(t=0)=10$, but with temperatures ranging between 15 and $35^{\circ} \mathrm{C}$ with an increment of $1^{\circ} \mathrm{C}$. M. quadripunctulatus mortality rate was assumed equal to the average of the values found in our experiments (reported in Table 1) because no appreciable temperature dependence was found over the first 22 days in the climatic chamber experiments.

While Figure 6A underlines the temperature sensitivity of infection progression as a function of time already noted in the experiments of Figure 3, Figure 6B highlights progression patterns for symptomatic plants $P_{t}$ substantially different from $P_{i}$ because the latency in plants $L_{P}(T)$ scales with a second power of $T$ (Figure 2). In particular, symptoms at temperatures $10^{\circ} \mathrm{C}$ and $35^{\circ} \mathrm{C}$ appeared later than at intermediate temperatures but evolved in substantially different ways, that is, slowly at $10^{\circ} \mathrm{C}$ and rapidly at $35^{\circ} \mathrm{C}$.

To characterize the epidemics time scales, the times $t_{50}, t_{75}$ and $t_{95}$ that the infection and symptoms took to appear in $50 \%, 75 \%$ and $95 \%$ of the plants were calculated. A comparison of $t_{50}, t_{75}$ and $t_{95} v s T$ showed that the infection involved an increasing fraction of plants in a monotonically shorter time for increasing $T$ (Figure 6C). Conversely, symptoms appeared in $50 \%, 75 \%$ and $95 \%$ of the plants in a time frame that followed a parabolic-like behavior, that is, decreasing with $T$ increasing above $15^{\circ} \mathrm{C}$ and increasing for $T$ beyond $25^{\circ} \mathrm{C}$. We also noted that the difference among $t_{50}, t_{75}$ and $t_{95}$ decreased nonlinearly from low to high temperatures [e.g., $\left(t_{95}-t_{50}\right)$ was smaller at high $T$ as compared to low $T$, Figure $6 \mathrm{D}]$. Finally, we noted an optimal temperature at around $25^{\circ} \mathrm{C}$ leading to the fastest epidemics progression, while any other temperatures resulted in a slower progression. This behavior can be ascribed to the combination of nonlinearly temperature-dependent latency $L_{P}$ and linearly temperature-dependence spreading rate $D$ '.

\section{Epidemics sensitivity to vector presence}

The epidemics progression in a close system such as in the climatic chamber experiments presented here is particularly susceptible to the initial number of adult vector insects of $M$. quadripunctulatus introduced in the cage because of the absence of migrating adults. The epidemics dynamics was therefore replicated as we mentioned abowe for $T$ ranging between 15 and $35^{\circ} \mathrm{C}$ and for an initial number of infective adults of $M$. quadripunctulatus ranging between 2 and 20. Results in Figure 7 highlights that $t_{50}$ of $C$. carinatum with symptoms decreased more than exponentially as $I_{t 0}=I_{t}(t=0)$ increased from 2 to 20 . We concluded from these simulations that the outbreak progression rate is strongly linked to the presence of infective vectors as prescribed by Eq. (1a).

\section{Discussion and Conclusions}

The dynamics of CYP epidemics in $C$. carinatum plants and its leafhopper vector $M$. quadripunctulatus have been investigated using an innovative controlled experimental approach that integrates, for the first time, the effect of temperature on several epidemiological parameters including latency in vector and host, insect mobility and transmission efficiency, and insect mortality rate. This approach provided results more reliable than experiments devoted to assess each single component of CYP epidemics. The modeling framework developed on the basis of these experimental data also represents the first attempt to predict the impact of temperature on phytoplasma epidemics progression. These experiments provided statistically significant results and, in conjunction with experiments devoted to assess each single component of the pathosystem, allowed us to develop and test a relatively simple modeling framework to analyze the epidemics progression in the temperature continuum.

In our modelling framework, the epidemics effective spreading rate, $D$ ', combines the insect mobility rate, $D$, with the probability $\beta$ for healthy plants to become infected after inoculation of CYP during insect feeding. Because our experiments and modeling results clearly show that $D$ 'increases with the temperature, we infer that also $D$ and $\beta$ change with the temperature. We can hypothesize two mechanisms to explain why $D^{\prime}$ increases with the temperature: (a) both $D$ and $\beta$ increase evenly with $T$, and (b) either $D$ or $\beta$ prevalently increases with $T$. We exclude, however, that one of the two parameters could decrease for $T$ increasing within the range of temperatures analyzed here. In both hypotheses, an increase in temperature may induce more frequent insect movement resulting in a higher number of visited plants. This would be reasonable as the metabolism of heterothermic insects, including flight behavior, is directly influenced by the temperature. Possibly, the feeding behavior may be susceptible to temperature, and
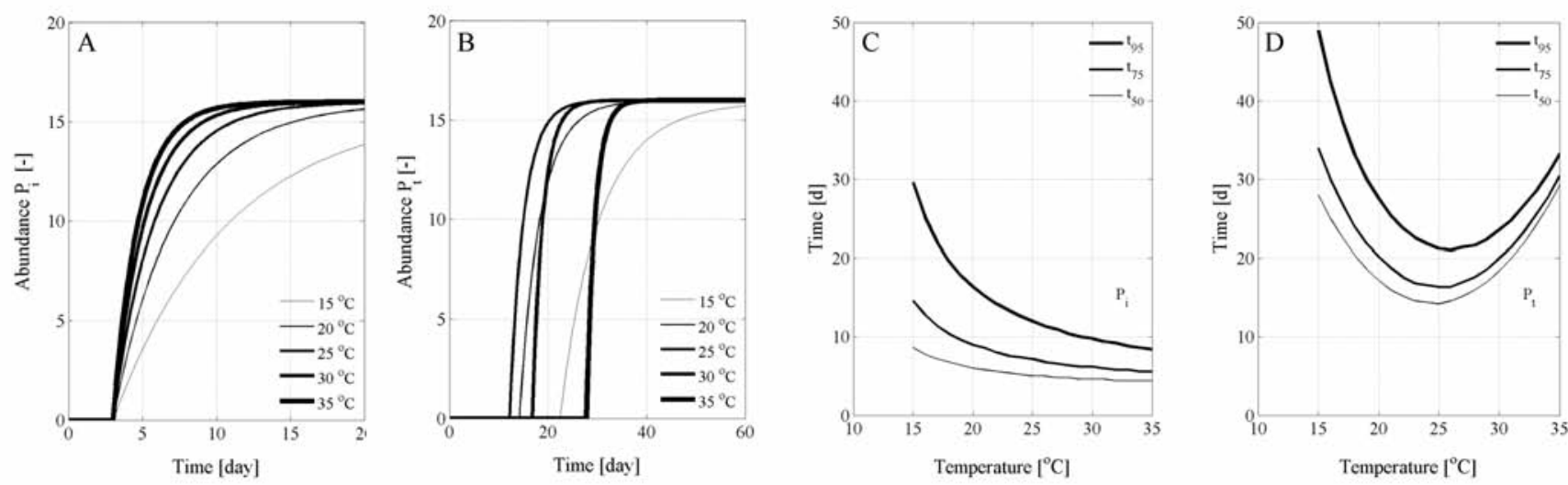

Figure 6. A) and B) number of infected and infective plants $P_{i}(t)$ and $P_{t}(t)$, respectively, for temperatures in the range $\left.15-35^{\circ} \mathrm{C} . \mathrm{C}\right)$ and D) elapsed time $t_{50}, t_{75}$ and $t_{95}$ that yield $50 \%, 75 \%$ and $95 \%$ of infected plants and infective plants, respectively, for the same range of temperatures. 
the probability $\beta$ could be influenced as well; phytoplasmas are phloemrestricted and persistently-transmitted pathogens during vector phloem ingestion. Insect feeding behavior includes probing in the parenchyma with short feeding probes, and phloem ingestion with longer feeding periods. How and if probing and feeding behaviors change with the temperature is not currently known but it is possible that a more active phloem ingestion occurs at higher temperatures, thus resulting in higher transmission probability $\beta$. Note also that we have implicitly assumed a uniform insect mobility rate $D$ regardless of gender, whereas male and female $M$. quadripunctulatus adults may move at different rates, thus with a potential effect on epidemics effective spreading rate $D$ '. Leafhopper males are considered more mobile than females (revised in Lessio \& Alma, 2004) but in experiments, carried out in our lab under the same experimental conditions of this work (unpublished data), M. quadripunctulatus males and females visited a similar number of plants.

More generally, the way $D$ and $\beta$ change with the temperature can not be independently assessed unless the mobility of individual insects is tracked and decoupled from the epidemics propagation in plants and insects. An analysis in this direction would ultimately clarify the effect of temperature on insect mobility and feeding behavior but would require experiments specifically designed for this aim, which was beyond the purpose of this work.

Finally, M. quadripunctulatus adult survival was not substantially affected by temperature in our experiments. One possible explanation is that the experimental time scale of 22 days was not long enough to highlight differences. In fact, experimental data in D'Amelio et al. (2008) show that $75 \%$ of $M$. quadripunctulatus male and female affected by CYP survived in average for more than 21 day at $25{ }^{\circ} \mathrm{C}$, data that are consistent with our finding.

The effect of various temperatures was analyzed in each parameters $\left(L_{l}, L_{P}, D, t_{s}\right.$ and $\left.\delta\right)$ and the epidemics progression curve as a whole. In the latter analyses, the temperature was assumed constant in each simulation whereas daily and seasonal variations coexist in real condi-

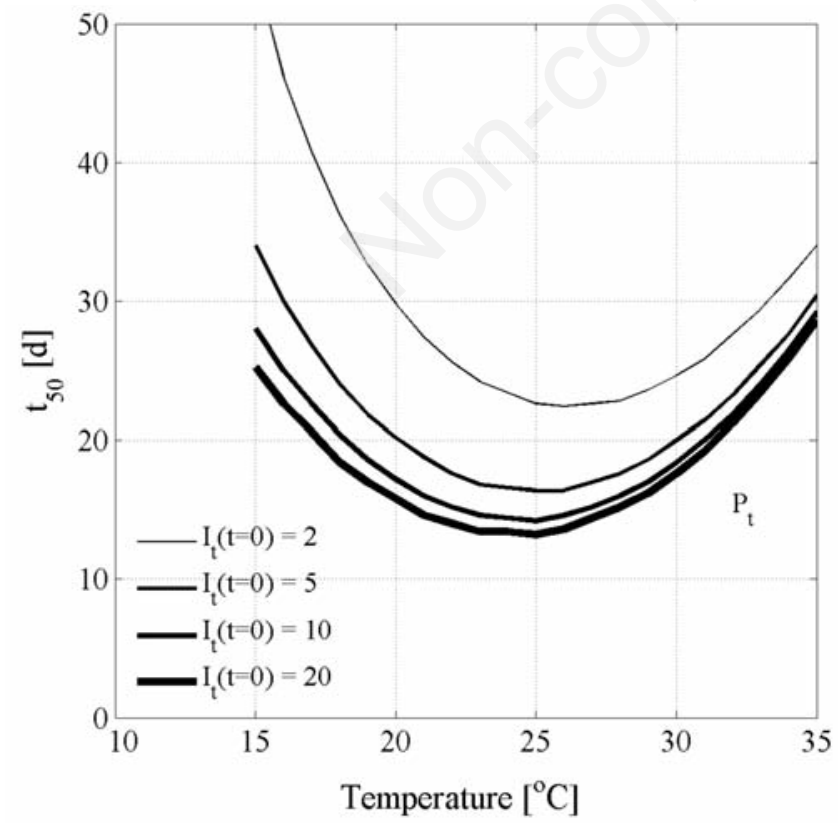

Figure 7. Elapsed time $t_{50}$ relative to symptomatic plants $P_{t}$ as a function of the temperature $T$ and for various initial conditions relative to infective vectors. tions. Clearly, this approach was the simplest method to test the effect of temperature on the epidemics progression curve; simulating actual daily temperature variations requires phytotrons with continuous temperature variations that were not available. Note that under actual field conditions, and besides fluctuating temperatures, several other factors directly and indirectly affect the epidemics including rainfall, and wind intensity and direction, while vector natural enemies and chemicals (e.g., insecticides) may act as disturbing factors. Inclusion of these factors in our model was beyond the purpose of this work, but we recognize the importance of assessing the effects of these factors for application to real conditions. For example, in an earlier work on the biological life-cycle of Scaphoideus titanus, the effect of stage timing, development rate and sinks due to biotic and abiotic factors were found to substantially affect if not determine the population presence over multiple sequential years (Maggi et al., 2013).

We remark here that while the findings of the experimental and modeling work can generally be applicable to plant-insect vector-phytoplasmas systems, there may exist cases of exception. For example, phytoplasmas are fully systemic in herbaceous hosts, like in our CYP-daisy experimental model (Saracco et al., 2006) and can analogously be described with the system of equations introduced in this work, which can also be used with systemic plant viruses. On the other hand, phytoplasmas may not be systemic in woody hosts (e.g., grapevine infected by Flavescence dorée); in such cases, only some branches of the plant may be affected, hence detecting the pathogen experimentally from plant tissues using PCR methods has to be carefully conducted based on visible symptoms. From a mathematical point of view, this aspect does not lead to any change in the structure of the equations, but may result in different acquisition probability because the likelihood that a leafhopper acquires the pathogen from an infectious plant is proportional to the fraction of plant that is affected (i.e., the entire plant when the pathogen is systemic, or less when the pathogen is non fully systemic). Pathogen acquisition was not part of the modeling work presented here, but we recognize that this aspect should be investigated in both systemic and non fully systemic plant pathogens in relation to the acquisition probability.

In conclusion, our experiments demonstrated that: i) CYP incubation and latency periods in plants and insects is nonlinearly dependent on temperature; ii) the epidemics effective spreading rate increases linearly with the temperature, while the pathogen detection time in plants decreases with the temperature. Scaling laws were derived from experiments and used in a newly-developed mechanistic model of CYP epidemics in vector insects and host plants, and demonstrated that: iii) the response of the epidemics progression curve within the temperature continuum is highly nonlinear and strongly depends on the sensitivity of the mentioned parameters to temperature.

We conclude that temperature must be included in mathematical representations of epidemics dynamics, especially when epidemiological projections are to be assessed within the framework of locally variable climatic conditions and in the framework of global climate warming. However, in evaluating the possible impact of global warming on phytoplasma diseases, we should consider that each phytoplasmaplant-vector association may be different and, to achieve reliable predictions of phytoplasma disease spread under real conditions, a number of phytoplasma-plant vector associations should be investigated, that could possibly lead to the design of a mathematical model or parameterization specific to phytoplasma diseases.

\section{Acknowledgements}

F. Maggi was partly supported by the 2010 Lagrange Fellowship, Fondazione CRT, Torino, Italy, and the ISI Foundation, Torino, Italy. 


\section{References}

BOSCO D., D’AMELIO R., 2010 - Transmission specificity and competition of multiple phytoplasmas in the insect vector. - In: WEINTRAUB P.G., JONES P., (eds). Phytoplasmas. CAB International, Wallingford, pp 293-308.

BOSCO D., GALETTO L., LEONCINI P., SARACCO P., RACCAH B., MARZACHÌ C., 2007 - Interrelationships between 'Candidatus Phytoplasma asteris' and its leafhopper vectors (Homoptera: Cicadellidae). - J. Econ. Entomol. 100: 1504-1511.

CONTI M., D’AGOSTINO G., CASETTA A., MELA L., 1988 - Some characteristics of chrysanthemum yellows disease. - Acta Horticulturae 234: 129-136.

D'AMELIO R., PALERMO S., MARZACHI C., BOSCO D., 2008 - Influence of Chrysanthemum yellows phytoplasma on the fitness of two of its leafhopper vectors, Macrosteles quadripunctulatus and Euscelidius variegatus. - Bull. Insectol. 61: 349-354.

DOHERTY J., 2004 - PEST model-independent parameter estimation. $5^{\text {th }}$ ed. Watermark Numerical Computing, Brisbane.

FERRISS R.S., BERGER P.H., 1993 - A stochastic simulation model of epidemics of arthropod-vectored plant viruses. - Phytopathology 83 : 1269-1278.

FOISSAC X., WILSON M., 2010 - Current and possible future distributions of phytoplasma diseases and their vectors. - In: WEINTRAUB P.G., JONES P., (eds). Phytoplasmas. CAB International, Wallingford, pp 309-324.

GALETTO L., BOSCO D., MARZACHÍ C., 2005 - Universal and group-specific Real Time PCR diagnosis of flavescence dorée (FD, 16Sr-V), bois noir (BN, 16Sr-XII) and apple proliferation (AP, 16Sr-X) phytoplasmas from field-collected plant hosts and insect vectors. - Ann. Appl. Biol. 147: 191-201.

IPCC, 2008 - IPCC Technical Paper VI - IPCC Secretariat, Geneva, 210 pp.

JEGER M.J., VAN DEN BOSCH F., MADDEN L.V., HOLT J., 1998 - A model for analysing plant-virus transmission characteristics and epidemic development. - IMA J. Math. Appl. Med. Biol. 15: 1-18.

LEE I.M., DAVIS R.E., GUNDERSEN-RINDAL D.E.. 2000 - Phytoplasma: phytopathogenic mollicutes. - Annu. Rev. Microbiol. 54: 221-255.

LEE I.M., GUNDERSEN-RINDAL D.E., DAVIS R.E., BOTTNER K.D., MARCONE C., SEEMUELLER E., 2004 - 'Candidatus Phytoplasma asteris' a novel phytoplasma taxon associated with aster yellow and related disease. - Int. J. Syst. Evol. Microbiol. 54: 1037-1048.

LEE I.M., GUNDERSEN D.E., HAMMOND R.W., DAVIS R.E., 1994 - Use of mycoplasmalike organism (MLO) group-specific oligonucleotide primers for nested-PCR assays to detect mixed MLO infection in a single host plant. - Phytopathology 84: 559-66.

LEE I.M., HAMMOND R.W., DAVIS R.E., GUNDERSEN D.E., 1993 Universal amplification and analysis of pathogen 16SrDNA for classification and identification of mycoplasmalike organisms. Phytopathology 83: 834-42.

LESSIO F., ALMA A., 2004 - Dispersal patterns and chromatic response of Scaphoideus titanus Ball (Homoptera Cicadellidae), vector of the phytoplasma agent of grapevine Flavescence dorée. - Agric. Forest Entomol. 6: 121-127.

MADDEN L.V., JEGER M.J., VAN DEN BOSCH F., 2000 -A theoretical assessment of the effects of vector-virus transmission mechanism on plant virus epidemics. - Phytopathology 90: 576-594.

MAGGI F., MARZACHI' C., BOSCO D., 2013 - A stage-structured model of Scaphoideus titanus Ball in Vineyards. - Env. Entomol. 42: 181-193.

MARZACHI' C., MILNE R.G., BOSCO D., 2004 - Phytoplasma-plant-vector relationships. - In: PANDALAI S.G., GAYATHRI A., (eds). Recent research development in plant pathology. Vol. 3. Research Signpost, Trivandrum, pp 211-241.

MURRAL D.J., NAULT L.R., HOY C.W., MADDEN L.V., MILLER S.A., 1996 - Effects of temperature and Vector Age on transmission of two Ohio strains of Aster yellows phytoplasma by the Aster Leafhopper (Homoptera: Cicadellidae). - J. Econ. Entomol. 89: 1123-1232.

PALERMO S., ARZONE A., BOSCO D., 2001 - Vector-pathogen-host plant relationships of chrysanthemum yellows (CY) phytoplasma and the vector leafhoppers Macrosteles quadripunctulatus and Euscelidius variegatus. - Entomol. Exp. Appl. 99: 347-354.

ROGGIA C., CACIAGLI P., GALETTO L., PACIFICO D., VERATTI F., BOSCO D., MARZACHI C. 2014 - Flavescence dorée phytoplasma titre in fieldinfected Barbera and Nebbiolo grapevines. - Plant Pathology 63: 31-41.

SALAR P., CHARENTON C., FOISSAC X., MALEMBIC-MAHER S., 2013 Multiplication kinetics of Flavescence dorée phytoplasma in broad bean. Effect of phytoplasma strain and temperature. - Europ. J. Plant Pathol. 135: 371-381.

SARACCO P., BOSC0 D., VERATTI F., MARZACHÌ C., 2006 Quantification over time of chrysanthemum yellows phytoplasma (16Sr-I) in leaves and roots of the host plant Chrysanthemum carinatum (Schousboe) following inoculation with its insect vector. Physiol. Mol. Plant Pathol. 67: 212-219.

VAN MAANEN A., XU X.M., 2003 - Modelling plant disease epidemics. Europ. J. Plant Pathol. 109: 669-682.

VANDERMEER J., POWER A., 1990 - An epidemiological model of the corn stunt system in Central America. - Ecol. Modelling 52: 235-248.

WEINTRAUB, P.G., BEANLAND, L.A., 2006 - Insect vectors of phytoplasmas. - Annu. Rev. Entomol. 51: 91-111. 